\title{
El alivio del dolor: un derecho humano universal
}

\author{
C. Martínez Caballero' ${ }^{1}$ F. Collado Collado ${ }^{2}$, J. Rodríguez Quintosa ${ }^{3}$ y J. Moya Riera ${ }^{4}$, on behalf of Efhre \\ International University, EMNIPRE Group (European Multidisciplinary Network in Pain, Research \\ and Education)
}

${ }^{1}$ Directora de Proyectos Científicos de EMNIPRE. Efhre International University. Belize. ${ }^{2}$ Clínica del Dolor. Unidad de Dolor Agudo. Hospital Puerta del Mar. Cádiz. ${ }^{3}$ President. Efhre International University. Belize. ${ }^{4}$ Director del Fórum Mediterráneo Multidisciplinar contra el Dolor. Fundador de la Asociación contra el Dolor "Sine Dolore"

Martínez Caballero C, Collado Collado F, Rodríguez Quintosa J, Moya Riera J, on behalf of Efhre International University, EMNIPRE Group (European Multidisciplinary Network in Pain, Research and Education). El alivio del dolor: un derecho humano universal. Rev Soc Esp Dolor 2015; 22(5): 224-230.

\begin{abstract}
Pain is one of the symptoms that more suffering produces in any disease, being a basic health problem worldwide. However, they often do not receive suitable treatment for cultural, religious, societal and health professionals attitudes as well as political and economic reasons.

From the ethical point of view relieve pain is a human right and an obligation for health professionals. The responsibility of professionals with regard to providing adequate pain control is contained in the Hippocratic Oath and the Declaration of $\mathrm{Ge}^{-}$ neva that guarantee the patients' health.

From a legislative perspective, international law on human rights forces signatories States to protect with all the resources at its disposal the guaranteed rights without expressly establish the right to adequate pain relief. However, the health right implies the right to an adequate pain management.

The article 43 of the Spanish Constitution of 1978 recognizes the right to health protection and therefore the right of citizens to receive adequate health care. Likewise, Article 43 includes a matter for the public authorities to organize and safeguard public health through preventive measures and the necessary benefits and services. Even without specifically contemplating the right to pain relief, an adequate health care cannot ignore an important issue as pain relief.
\end{abstract}

The World Health Organization (WHO), has stated that pain relief is a fundamental right and that incurs in a severe lack of ethic of the health professional who prevents a human from accessing the possibility of pain alleviation. WHO has issued the necessary criteria for a good pain control among which includes appropriate training of health professionals and the availability of drugs for the treatment of pain.

The attitude or behavior that neglects something or neglected something is called negligence. They might as well be called situations where there are enough means to perform analgesic therapy and yet it is not done. The reasons for this situation of therapeutic negligence include reluctance to prescribe opioids due to the fear of the undesirable effects of them, prescribing a lower analgesic doses, prescribing drugs at intervals of exceeding the recommended dosage or is demand patterns, delivery by nurses of less doses of the prescribed drug believing that they should not administer the treatment when the patient does not have pain and finally resignation of patients to suffer pain.

Key words: Pain relief. Pain management. Human right.

\section{RESUMEN}

El dolor es uno de los síntomas que más sufrimiento produce en cualquier enfermedad y constituye un problema básico de salud en todo el mundo. Sin embargo, con frecuencia no recibe el tratamiento adecuado por razones culturales, religiosas, actitudes de la sociedad y de los profesionales sanitarios, así como por motivos políticos y económicos.

Desde el punto de vista ético, aliviar el dolor es un derecho del ser humano y una obligación de los profesionales sanitarios. La responsabilidad de los profesionales en lo que respecta a proporcionar un control adecuado del dolor está recogida en el Juramento Hipocrático y en la Declaración de Ginebra, que exige ante todo velar por la salud del paciente.

Desde un punto de vista legislativo, la legislación internacional en materia de derechos humanos obliga a los Estados firmantes a proteger con todos los recursos a su alcance los de- 
rechos que la misma garantiza, sin establecer expresamente el derecho a un alivio adecuado del dolor. Sin embargo, el derecho a la salud lleva implícito el derecho a un adecuado tratamiento del dolor.

La Constitución Española de 1978, en su artículo 43, reconoce el derecho a la protección de la salud y por tanto el derecho de los ciudadanos a recibir una asistencia sanitaria adecuada. Así mismo, el artículo 43 incluye que compete a los poderes públicos organizar y tutelar la salud pública a través de medidas preventivas y de las prestaciones y servicios necesarios. Aun no contemplando expresamente el derecho al alivio del dolor, garantiza una adecuada asistencia sanitaria que no puede dejar de lado un tema tan importante como es el tratamiento del dolor.

La Organización Mundial de la Salud (OMS) determinó que el alivio del dolor es un derecho fundamental y que incurre en una falta de ética grave aquel profesional de la salud que impida a un ser humano el acceso a la posibilidad de alivio del mismo. La OMS ha dictado los criterios indispensables para un buen control del dolor, entre los que recoge la adecuada formación de los profesionales de la salud y la disponibilidad de fármacos para el tratamiento del dolor.

La actitud o comportamiento del que descuida algo o se descuida en algo se denomina "negligencia". Se podrían denominar así situaciones en las que no se realiza una terapéutica analgésica adecuada existiendo medios suficientes para hacerlo. Entre las razones que explican esta situación de "negligencia terapéutica" se encuentra la reticencia a prescribir fármacos opioides debido a un miedo desmesurado a los efectos indeseables de los mismos, la prescripción a dosis inferiores a las analgésicas, a intervalos de dosificación superiores a los recomendados o en pautas a demanda, la administración de menos dosis de los fármacos prescritos por parte de Enfermería en la creencia errónea de que no deben suministrarse los analgésicos pautados cuando el enfermo se encuentra sin dolor y la resignación de los enfermos a sufrir dolor.

Palabras clave: Alivio del dolor. Manejo del dolor. Derecho humano.

\section{INTRODUCCIÓN}

Desde los albores de la humanidad el dolor y el deseo de controlarlo han acompañado al ser humano a lo largo del tiempo. El dolor es uno de los síntomas que más sufrimiento produce en cualquier enfermedad, constituyendo un problema básico de salud en todo el mundo. Sin embargo, con frecuencia no recibe el tratamiento adecuado por razones culturales, religiosas, actitudes de la sociedad y de los profesionales sanitarios, así como por motivos políticos y económicos.

La International Association for the Study of Pain (IASP) define el dolor como "una experiencia sensorial y emocional desagradable asociada con una lesión real o potencial de los tejidos, u ocasionada por dicha lesión" (1). Esta definición reconoce la existencia de dos componentes del dolor, uno nociceptivo o sensorial, que constituye la sensación dolorosa y se debe a la transmisión de los impulsos por las vías nerviosas hasta el córtex cerebral, y otro afectivo que constituye la vivencia del dolor y está relacionado con factores culturales, sociales, emocionales y psicológicos.

Atendiendo a la evolución temporal, el dolor se clasifica en crónico y agudo. El dolor agudo es una señal de alarma como consecuencia de un daño tisular somático o visceral y desaparece generalmente con la reparación de la lesión que lo originó. Sin embargo, un tratamiento inadecuado puede llevar a la cronificación del mismo. El dolor crónico, por el contrario, no posee una función biológica de protección y conlleva alteraciones físicas, emocionales y sociales que afectan la calidad de vida del paciente.

Aliviar el dolor es un derecho del ser humano y una obligación ética de los profesionales sanitarios. El Artículo 25 de la Declaración Universal de los Derechos Humanos (2) hace énfasis en el derecho a un nivel adecuado de salud y bienestar, llevando implícito el derecho al adecuado tratamiento del dolor.

\section{EL ALIVIO DEL DOLOR DESDE UN PUNTO DE VISTA ÉTICO}

La ética es la parte de la Filosofía que estudia la bondad o la malicia intrínseca de los actos y de las conductas humanas. La Medicina desde sus inicios ha estado relacionada con la ética, ya que su objetivo ha sido siempre conseguir el bien del ser humano enfermo (3).

El término "bioética" procede del griego bios (vida) y ethos (comportamiento, costumbre). El objetivo principal de la bioética es, por tanto, el estudio de la relación existente entre la vida y los principios o pautas de la conducta humana. La bioética en el campo de la Medicina se basa en los cuatro principios formulados por Beauchamp y Childress (4): beneficencia, no maleficencia, autonomía y justicia. El principio bioético más elemental y sobre el que se basan los enumerados anteriormente es el respeto a la dignidad de la persona basado en un enfoque integral y humanista de la asistencia sanitaria.

La responsabilidad de los profesionales en lo que respecta a proporcionar un control adecuado del dolor está recogida en el Juramento Hipocrático (5) y en la Declaración de Ginebra (6), que exige ante todo velar por la salud del paciente. Asímismo, el Código Internacional de Ética Médica (7) estipula que el médico debe a sus pacientes toda su lealtad y todos los recursos científicos disponibles para ellos.

El alivio del dolor es un claro ejemplo del principio de beneficencia. Este principio, fundamento del Juramento Hipocrático, consiste en orientar el ejercicio de la Medicina a buscar el bien del paciente. Permitir que sufra dolor sin poner los medios necesarios para evitarlo vulnera abier- 
tamente este principio. No proporcionar los medios terapéuticos disponibles actualmente para el alivio del dolor constituye una forma de abandono que, llevado a un caso extremo, se podría considerar como omisión del deber de socorro. Evitar el dolor y el sufrimiento es la primera obligación moral y profesional no solo del médico, sino de todo el personal sanitario implicado en el cuidado del paciente. Por ello, permitir que el paciente experimente dolor por no utilizar las medidas terapéuticas disponibles es una práctica medica inmoral que vulnera el principio de beneficencia y constituye una de las más escandalosas negligencias médicas.

El principio de no maleficencia se basa en el principio hipocrático de primum non nocere, es decir, "ante todo no hacer daño". Según este principio ningún profesional sanitario debería ocasionar perjuicios a un enfermo, valorando siempre el balance entre los beneficios y los riesgos de toda actuación terapéutica. Sin embargo, hay situaciones en las que no es posible evitar un trauma al paciente dado su carácter cruento, como ocurre en las intervenciones quirúrgicas o en ciertas pruebas diagnósticas de carácter invasivo. En estos casos, el no poner los medios necesarios para evitar o minimizar en la medida de lo posible el dolor producido por la intervención diagnóstica o terapéutica es actuar en contra del principio de no maleficencia.

El principio de autonomía se refiere a la capacidad que tiene el enfermo para decidir sobre su tratamiento. Al contrario que los anteriores, es un principio que siempre ha estado ausente de la tradición médica, a pesar de la gran importancia que ha adquirido en los últimos años. Durante siglos el paciente nunca ha participado en la toma de decisiones y el médico era quien decidía. El deber del médico era hacer el bien al paciente y el del paciente aceptarlo (8). El enfermo, por el mero hecho de serlo, carecía de capacidad para elegir. En la actualidad, desde el punto de vista ético, se plantea la importancia de que el paciente participe activamente en la elección del tratamiento tras recibir información suficiente y adecuada. No proporcionar información sobre las medidas disponibles para el control del dolor contraviene el derecho del paciente de participar activamente en la elección de la terapéutica. Impedir su participación afecta seriamente al principio de autonomía. Sin embargo, el paciente debe participar en la toma de decisiones de una manera racional, no dejándose influenciar por el desconocimiento, por ideas irracionales ni por presiones externas.

Este principio es sumamente importante porque obliga a informar al paciente, si así lo desea, sobre el diagnóstico, el pronóstico y las posibilidades terapéuticas, con sus riesgos y beneficios. Permite al enfermo rechazar todo tipo de tratamiento o elegir uno distinto al propuesto. Debería permitir al paciente, dentro de lo posible y con las limitaciones legales vigentes, elegir el momento, lugar y forma de su muerte. Para promover este principio se ha propugnado el llamado "consentimiento informado", como forma de garantizar que el paciente ha recibido una información adecuada sobre el acto médico que se va a aplicar y que manifiesta su acuerdo. El consentimiento informado es tanto más importante cuanto mayores sean los riesgos del tratamiento propuesto o de la técnica de exploración a la que se someta y cuantas más posibilidades existan de fracaso.

Según el principio de justicia la Medicina debe tratar a todos los seres humanos sin ningún tipo de discriminación, debiendo proteger más a los más desfavorecidos. El bien común o el bien de todos deben ser siempre superiores al bien de individuos concretos (9). Este principio se basa en dos hechos: el primero de ellos es que todas las personas, por el mero hecho de serlo, tienen la misma dignidad, independientemente de cualquier circunstancia, y por tanto son merecedoras de igual consideración y respeto; el segundo hecho es que hay que luchar por una distribución justa y equitativa de los siempre limitados recursos sanitarios para conseguir el máximo beneficio en la comunidad, evitando desigualdades en la asistencia sanitaria. Este principio convierte al personal sanitario en gestor y administrador de los recursos y de los servicios, que deberá utilizar de una forma efectiva y eficiente, evitando actuaciones sanitarias inadecuadas.

\section{EL ALIVIO DEL DOLOR DESDE UN PUNTO DE VISTA LEGISLATIVO}

La legislación internacional en materia de derechos humanos se articula en la Declaración Universal de Derechos Humanos (1948) (2), el Pacto Internacional de Derechos Civiles y Políticos (1966) (10) y el Pacto Internacional de Derechos Económicos, Sociales y Culturales (1976) (11). La Declaración Universal de los Derechos Humanos, en su artículo 25, establece el derecho a un nivel adecuado de salud y bienestar. El Comité de Derechos Económicos, Sociales y Culturales (CESCR) recoge en este pacto el derecho de toda persona al disfrute del más alto nivel de salud física y mental. Esta legislación obliga a los Estados firmantes a proteger con todos los recursos a su alcance los derechos que la misma garantiza, sin establecer expresamente el derecho a un alivio adecuado del dolor. Sin embargo, el derecho a la salud lleva implícito el derecho a un adecuado tratamiento del dolor.

La Constitución Española de 1978 (12), en su artículo 43 , reconoce el derecho a la protección de la salud y por tanto el derecho de los ciudadanos a recibir una asistencia sanitaria adecuada. Asímismo, el artículo 43 incluye que compete a los poderes públicos organizar y tutelar la salud pública a través de medidas preventivas y de las prestaciones y servicios necesarios. Aun no contemplando expresamente el derecho al alivio del dolor, garantiza una adecuada asistencia sanitaria que no puede dejar de lado un tema tan importante como es el tratamiento del dolor. 
En España, el derecho de autonomía del paciente, recogido en la ley 41/2002 (13) básica reguladora de la autonomía del paciente y de deberes y obligaciones en materia de información y documentación clínica, reconoce el derecho de los mismos a ser informados de todos lo aspectos de su enfermedad así como de los posibles tratamientos, con el fin de poder tomar decisiones sobre su propio cuidado. Esto incluye que los profesionales realicen un esfuerzo razonable para proporcionar un adecuado alivio del dolor, permitiendo al paciente tomar decisiones a este respecto.

\section{EL PAPEL DE LA OMS}

Hace algunos años la Organización Mundial de la Salud determinó que el alivio del dolor es un derecho fundamental y que incurre en una falta de ética grave aquel profesional de la salud que impida a un ser humano el acceso a la posibilidad de alivio del mismo (14).

La OMS ha dictado los criterios indispensables para un buen control del dolor:

- Educación de los profesionales de la salud, el público en general y administradores hospitalarios en el control del dolor.

- Disponibilidad de fármacos y un sistema de distribución eficiente y seguro.

- Cambios en las legislaciones y regulaciones que supriman las disposiciones que interfieren con el uso adecuado de opioides potentes.

La OMS publica en 1986 en Ginebra el documento denominado Cancer Pain Relief (Alivio del dolor en el cáncer) (15), recogido en 1998 en la revista Anesthesia \& Analgesia y que se conoce como "escalera analgésica de la OMS", con el objetivo de mejorar el tratamiento del dolor oncológico. Desde su creación y divulgación en el año 1986 se han producido modificaciones del mismo, algunas recogidas en la segunda edición del documento, de 1996 (16). Su estructura básica no se ha modificado, pero algunos de los medicamentos inicialmente propuestos han desaparecido o han sido relegados a un segundo plano. En el año 2006 se celebró el 20 aniversario de la publicación y puesta en marcha de la escalera analgésica de la OMS (17). Se ha evaluado, debatido su origen, su vigencia, sus éxitos y limitaciones, reconociéndose que, a pesar de ellas, su difusión ha mejorado de forma sustancial el tratamiento del dolor.

Este concepto, propuesto hace más de 20 años para el dolor oncológico con el objetivo de mejorar el uso de opioides sobre todo en países donde no estaban fácilmente disponibles, se extendió también al dolor no oncológico. Lo que en un principio constituyó una ayuda se convirtió con el tiempo en un obstáculo para el manejo adecuado del dolor. El concepto de "escalera analgésica" no se adapta a algunas situaciones, como ocurre con el dolor postoperato- rio. Luis Miguel Torres, en 2002, propone sustituir el concepto de "escalera analgésica" por "ascensor analgésico", en el cual el paciente dispone de cuatro botones para pulsar con diferentes opciones analgésicas según el dolor sea leve, moderado, severo o insoportable (18). Este sistema proporciona inmediatez en la respuesta analgésica, ya que no sería ético hoy en día mantener a un paciente con dolor mientras recorre los peldaños de la escalera de la OMS hasta instaurar el tratamiento con el fármaco adecuado.

\section{BASES DEL MANEJO INADECUADO DEL DOLOR Y CONCEPTO DE NEGLIGENCIA TERAPÉUTICA}

Numerosos estudios, llevados a cabo en los últimos años, indican que incluso en los casos en los que se trata el dolor el tratamiento es inadecuado o insuficiente (19). B. Ferrel, experta en el tratamiento del dolor, a menudo habla del efecto "triple-whammy": el médico prescribe por debajo de lo necesario, las enfermeras administran menos de lo prescrito y los pacientes no comunican todo su dolor (20).

La actitud o comportamiento del que descuida algo o se descuida en algo se denomina "negligencia" (21). Se podrían denominar así situaciones en las que no se realiza una terapéutica analgésica adecuada cuando existen medios suficientes para hacerlo. Entre las razones que explican esta situación de negligencia terapéutica se encuentra la gran reticencia a prescribir fármacos opioides (22) debido a la existencia de un miedo desmesurado a los efectos indeseables de los mismos, la prescripción a dosis inferiores a las analgésicas, a intervalos de dosificación superiores a los recomendados o en pautas a demanda, la administración de menos dosis de los fármacos prescritos por parte de Enfermería en la creencia errónea de que no deben suministrarse los analgesicos pautados cuando el enfermos se encuentra sin dolor y la resignación de los enfermos a sufrir dolor (23).

\section{ACTITUDES Y FORMACIÓN DE LOS PROFESIONALES SANITARIOS}

La falta de una formación adecuada sobre el manejo del dolor por parte del personal sanitario es una de las causas de la ausencia de evaluación o valoración inadecuada de la intensidad del dolor, así como de un tratamiento subóptimo del mismo. El desconocimiento de la fisiopatología del dolor y de la farmacocinética y farmacodinamia de los analgésicos es, en muchos casos, la causa de un tratamiento no adecuado, así como la opinión que tienen muchos profesionales de la salud de que el dolor debe soportarse lo máximo posible.

Entre los conceptos erróneos derivados de una formación inapropiada o insuficiente destacan la preocupación 
por la tolerancia, la dependencia y la adicción a los opiodes, ansiedad sobre el uso de opioides y sus posibles efectos secundarios (24), la teoría de que la analgesia dificulta el diagnóstico y la creencia de que la dosis de opioides debe relacionarse con la gravedad de la enfermedad en lugar de con la intensidad del dolor.

La comunicación inadecuada entre los profesionales sanitarios y los pacientes es otra de las razones del infratratamiento o mal tratamiento del dolor. Con frecuencia, y debido a distintas causas, no se estabece una comunicación fluida con el paciente, produciéndose lagunas en el cuidado del mismo como la falta de valoración rutinaria de la intensidad del dolor.

\section{RAZONES SOCIALES Y CULTURALES}

Las actitudes de los médicos son, a menudo, reflejo de las actitudes erróneas de la sociedad en general (25-27). Estos incluyen la inevitabilidad del dolor, no comunicar que se tiene dolor con el deseo de ser un buen paciente y esperar hasta que el dolor sea agudo antes de utilizar los opioides. Las actitudes sociales para aliviar el dolor están influenciadas por los conceptos prevalecientes sobre el dolor y su significado para el individuo y la sociedad (28). En las sociedades primitivas el dolor estaba relacionado con los malos espíritus o demonios en el cuerpo. Más tarde, y todavía hoy en algunas culturas, el dolor era un castigo y constituía una forma de expiación de los pecados.

Existen creencias populares debidas al desconocimiento de los avances de la Medicina en el terreno del tratamiento de dolor tanto oncológico como no oncológico. Este aspecto, llamado "asimetría de la información", puede llevar a que existan falsos conceptos o mitos respecto a que padecer dolor es un hecho normal. La visión que el paciente tiene sobre el uso de ciertos fármacos para el tratamiento del dolor puede estar influenciada por determinados conceptos, como que los fármacos para el alivio del dolor producen adicción e intolerables efectos secundarios.

La ausencia de información o educación del paciente sobre las consecuencias del dolor no tratado de forma adecuada y sobre las posibilidades de analgesia no solo no facilitan el manejo adecuado del dolor, sino que crean importantes barreras que dificultan el correcto abordaje del mismo.

\section{ACTITUDES RELIGIOSAS}

La religión ha cargado de significado el concepto de dolor (26). En muchas religiones la presencia de dolor se ha incorporado en la misma dimensión espiritual de la condición humana, tratando de lograr la trascendencia. Sin embargo, en la actualidad las religiones no ponen ningún impedimento para el control del dolor de los pacientes.

En la antigüedad se pensó que el dolor del hombre era un castigo por sus pecados. La Pasión de Jesucristo viene a dar un sentido nuevo al dolor. A partir de la Cruz, el sufrimiento se vuelve redentor. Para el cristianismo el dolor no es el castigo de una culpa, sino una oportunidad de purificación. La noción de que el dolor sirve como mecanismo de expiación y borra la culpa de los pecados está ampliamente difundida en el pensamiento católico.

El dolor está presente también en la Biblia. "Parirás con dolor", con esta frase enuncia el primer libro bíblico el castigo proferido por Dios a la mujer. El mandato divino condena así a la mujer occidental a parir con dolor y temor. La sentencia profética ha impactado en nuestra cultura y se ha popularizado hasta el punto que cuando atravesamos por una situación difícil y dolorosa decimos "¡esto es un parto!". Dar a luz siempre se han relacionanado con dolor y hasta no hace mucho tiempo era algo evidente que para parir siempre había que sufrir.

El Papa Benedicto XVI constata que la realidad del sufrimiento es una realidad que forma parte de la existencia humana. Sin embargo, afirma que debemos hacer todo lo que esté dentro de nuestras capacidades y posibilidades para superar el sufrimiento.

\section{ACTITUDES POLÍTICAS}

En ocasiones existen barreras políticas y legales para el control eficaz del dolor. Los complejos problemas gubernativos varían de un país a otro, pudiendo incluir baja prioridad en lo que respecta al apoyo de los cuidados paliativos y Unidades de Tratamiento del Dolor, problemas en la disponibilidad de opioides y limitaciones para la prescripción de los mismos $(29,30)$.

\section{CONCLUSIÓN}

El dolor se puede considerar como la preocupación sanitaria más importante del mundo. El alivio del mismo debería constituir uno de los derechos fundamentales de los seres humanos. Sin embargo, el dolor no recibe un tratamiento suficiente ni adecuado; de hecho, siguen existiendo barreras para un control eficaz del mismo, barreras culturales, educativas, políticas y económicas.

Todavía no se han superado los obstáculos que impiden o dificultan el adecuado tratamiento del dolor. La mayoría de los pacientes continúan recibiendo un tratamiento analgésico que no refleja los avances de la últimas décadas. Esto conlleva un sufrimiento injustificado y supone un incremento del riesgo de aparición de complicaciones, así como un aumento de la morbilidad. 
En un futuro no muy lejano el alivio del dolor debería ser una realidad universal. Para ello sería necesaria la implantación en todos los hospitales de Unidades de Tratamiento del Dolor que posibiliten el tratamiento multidisciplinar del mismo y que se establezcan programas de coordinación con los Centros de Salud. Cambiar la actitud desde la Atención Primaria es fundamental para hacer más accesible el tratamiento del dolor.

A la necesidad de generalizar las Unidades de Dolor y los equipos de cuidados paliativos en número suficiente se debe unir un esfuerzo en la preparación de los profesionales de todas las especialidades, con el fin de eliminar la presencia del dolor como algo habitual. Una formación adecuada posibilitará que los médicos de Atención Primaria puedan tratar a sus pacientes o remitirlos a las Unidades de Dolor cuando ello sea necesario.

\section{EFHRE INTERNATIONAL UNIVERSITY, EMNIPRE GROUP (EUROPEAN MULTIDISCIPLINARY NETWORK IN PAIN, RESEARCH AND EDUCATION)}

Luis Aliaga Font. Clínica del Dolor. Hospital Quirón Teknon. Barcelona

Alicia Alonso Cardaño. Anestesióloga. Unidad de Dolor. Hospital San Pedro. La Rioja

Martín Carpintero Porrero. Anestesiólogo. Unidad de Dolor. Hospital de Cabueñes. Gijón

Rafael Cobos Romana. Director Científico del Master de Acupuntura Universidad Pablo de Olavide, Sevilla. Presidente de la Sociedad De Acupuntura Médica de España (SAME). Cofundador del Instituto para el Estudio y Tratamiento Integral del Dolor (IETID). Vocal de la Junta Directiva de la Asociación Andaluza del Dolor.

Francisco Collado Collado. Clínica del Dolor. Unidad de Dolor Agudo. Hospital Puerta del Mar. Cádiz

Manuel Corral Rosado. Coordinador Anestesiología Red Asistencial Juaneda. Menorca.

Gonzalo Cutillas Hernández. Coordinador Unidad de Dolor. Hospital Vithas Perpetuo Socorro. Alicante

Raúl Fernández Moya. Diplomado en Enfermería. Secretary General. Efhre International University.

Jordi Folch Ibáñez. Adjunto Servicio Anestesia. Reanimación y Patología del Dolor,.Hospital Plató. Barcelona

Andrés Glenny Faggi. Médico Adjunto Servicio de Oncología y Unidad de Dolor. Subdirector Médico. Policlínica Miramar. Palma de Mallorca

Jordi Guitart Vela. Jefe de Servicio de Anestesia, Reanimación y Patología del Dolor. Hospital Plató. Barcelona

Marian Lorente Gascón. Directora del Laboratorio de Anatomía Quirúrgica, Universidad Internacional de Catalunya.

Carmen Martínez Caballero. Clínica MEDart. Madrid. Directora de Proyectos Científicos de EMNIPRE. Efhre International University.
Pedro Mir Orfila. Médico estomatólogo. Cirujano Bucal. Menorca

Xavier Mir Mari. Odontólogo. Máster en Cirugía Bucal. Barcelona

Jordi Moya Riera. Director del Fórum Mediterráneo Multidisciplinar contra el Dolor. Fundador de la Asociación contra el Dolor "Sine Dolore".

Francisco Rodelas Montalvo. Unidad de Dolor. Consorci Sanitari del Garraf. Sant Pere de Ribas. Barcelona

Jaime Rodríguez Quintosa. President. Efhre International University

Manuel Jesús Sánchez del Águila. Coordinador de la Unidad de Dolor. Hospital Costa del Sol. Marbella

Juan Manuel Vaca Miguel. Clínica de Dolor. Valladolid

M. ${ }^{a}$ Isabel Vargas Domingo. Unidad de Dolor. Servicio de Anestesiología. Coordinadora del Área Quirúrgica del Parc Sanitari Sant Joan de Déu. Sant Boi de Llobregat

Domingo Ventura Vargas. Unidad de Dolor. Servicio Anestesiología, Reanimación y Terapia del Dolor. Hospital Universitario Puerto Real. Cádiz. Unidad de Tratamiento del Dolor Hospital Viamed Santa Angela de la Cruz. Sevi1la. Cofundador del Instituto para el Estudio y Tratamiento Integral del Dolor (IETID).

Alfonso Vidal Marcos. Director Unidad de Dolor. Hospital Sur. Grupo IDCSALUD. Alcorcón, Madrid

Xavier Vila Justrivo. Unidad de Dolor. Hospital Universitario Arnau de Vilanova. Lérida

Montserrat Viñals Gigena. Coordinadora de la Unidad de Dolor Crónico. Policlínica Nuestra Señora del Rosario. Ibiza

\section{DEDICATORIA}

Dedicado por todos los miembros de EMNIPRE al Dr. Luis Aliaga Font en recuerdo a su dedicación, entrega y profesionalidad, así como por su valiosa aportación a este grupo de investigación.

\section{CORRESPONDENCIA:}

Jordi Moya Riera

Fórum Mediterráneo Multidisciplinar contra el Dolor

e-mail: jordimoyariera@gmail.com

\section{BIBLIOGRAFÍA}

1. Merskey H. Pain terms: a list with definitions and notes on usage. Recommended by IASP Subcomitte on Taxonomy. Pain 1979;6:249.

2. Declaración Universal de los Derechos Humanos. http:// www.un.org/es/documents/udhr. Último acceso 11 noviembre 2014 . 
3. Azulay Tapiero A. Los principios bioéticos: ¿se aplican en la situación de enfermedad terminal? An Med Interna 2001;18(12):650-4.

4. Beauchamp TL\&Childress J. Principles of Biomedical Ethics. 5th ed. Oxford University Press; 2001.

5. Juramento Hipocrático. Universidad de Navarra. Facultades de Medicina, Ciencias y Farmacia. Departamento de Humanidades Biomédicas. Centro de Documentación de Bioética. http://www.unav.es/cdb/juramento1.html. Último acceso 11 noviembre 2014.

6. Declaración de Ginebra de la Asociación Médica Mundial. Universidad de Navarra. Facultades de Medicina, Ciencias y Farmacia. Departamento de Humanidades Biomédicas. Centro de Documentación de Bioética http://www.unav.es/ cdb/ammginebra1.html. Último acceso 11 noviembre 2014.

7. Código Internacional de Ética Médica. Universidad de Navarra. Facultades de Medicina, Ciencias y Farmacia. Departamento de Humanidades Biomédicas. Centro de Documentación de Bioética http://www.unav.es/cdb/ ammlondres1.html. Último acceso 11 noviembre 2014.

8. Sanz Ortiz J. Bioética y medicina paliativa. En: Gómez Sancho M. Medicina Paliativa en la Cultura Latina. Madrid: Arán Ed. 1999.p. 1161-76.

9. Gracia D. Fundamentos de Bioética. Madrid: Ed. Eudema. 1989.p. 202.

10. Pacto Internacional de Derechos Civiles y Políticos. (1966). http://www.acnur.org/t3/fileadmin/scripts/doc. php?file=biblioteca/pdf/0015. Último acceso 11 noviembre 2014.

11. Pacto Internacional de Derechos Económicos, Sociales y Culturales (1976). Oficina de Alto Comisionado para los Derechos Humanos. http://www.ohchr.org/SP/ProfessionalInterest/Pages/CESCR.aspx. Último acceso 11 noviembre 2014.

12. Constitución Española. Artículo 43. https://www.boe.es/ buscar/act.php?id=BOE-A-1978-31229. Último acceso 11 noviembre 2014.

13. Ley de Autonomía del Paciente 41/2002 de 14 de noviembre, básica reguladora de la autonomía del paciente y de deberes y obligaciones en materia de información y documentación clínica.
14. http://www.who.int/es. Último acceso 11 noviembre 2014.

15. World Health Organization. Cancer Pain Relief. Geneve; 1986.

16. World Health Organization. Cancer Pain Relief. 2nd ed. Geneve; 1996.

17. World Health Organization. Cancer Pain Relief. Geneve; 2006.

18. Torres LM, Calderón E, Pernia A, et al. De la escalera al ascensor. Rev Soc Esp Dolor 2002;9:289-90.

19. Soler E, Montaner MC. Consideraciones bioéticas en el tratamiento del dolor. Persona y bioética 2004;8(20-21):49-69.

20. Kennedy-Schwarz J. Ethical issues. AJN 2000;100:8-9.

21. Moliner M. Diccionario de uso del español. Madrid. Aguilar 1979.

22. Morgan JP. American opiophobia customany underutilization of opioids analgesics. En: Stimmel B, editor. Controversies in Alcoholism and Substance Abuse. Nueva York: Howarth Press; 1986.p. 163-73.

23. Baños JE, Bosch F. Conceptos generales de algesiología. En: Aliaga L, Baños JE, editor. Tratamiento del dolor, teoría y práctica. Barcelona: Plublicaciones Permanyer; 2002.p. $1-8$.

24. BennetDS, Carr DB. Opiophobia as a barrier to the treatment of pain. J Pain Palliative Care Pharmacother 2002; 16(1):105-9.

25. Morris DB. The culture of pain. Berkley, CA: University of California Press; 1991.

26. Morris DB. Illness and culture in the postmodern age. Berkley, CA: University of California; 1998.

27. Papper EM. Romance, poetry and surgical sleep. Literature influences medicine. Westport, CT: Greenwood Press; 1995.

28. Procacci P, Maresca M. Pain concept in Western civilization: a historical review. En: Benedetti $\mathrm{C}$ et al, editores. Advances in pain research and therapy. New York: Raven Press; 1984.

29. Editorial. Pain relief: a universal human right. Pain 2004; 112:1-4.

30. Brennan F, Carr DB. Pain management: a fundamental human right. Pain Medicine 2007;105(1):205-21. 